\title{
Patient motivations for non-adherence to lung cancer screening in a military population
}

\author{
Kenneth P. Seastedt ${ }^{1}$, Michael J. Luca ${ }^{2}$, Jared L. Antevil ${ }^{3}$, Robert F. Browning ${ }^{4}$, Philip S. Mullenix ${ }^{5}$, \\ Junewai L. Reoma ${ }^{5}$, Sean A. McKay ${ }^{2}$ \\ ${ }^{1}$ Department of Surgery, ${ }^{2}$ Department of Pulmonology, USUHS F. Edward Hébert School of Medicine, Bethesda, MD, USA; ${ }^{3}$ Department \\ of Cardiothoracic Surgery, Veterans Affairs Medical Center, Washington, DC, USA; ${ }^{4}$ Department of Pulmonology, Division of Interventional \\ Pulmonology, ${ }^{5}$ Department of Cardiothoracic Surgery, USUHS F. Edward Hébert School of Medicine, Bethesda, MD, USA \\ Contributions: (I) Conception and design: All authors; (II) Administrative support: All authors; (III) Provision of study materials or patients: All \\ authors; (IV) Collection and assembly of data: All authors; (V) Data analysis and interpretation: All authors; (VI) Manuscript writing: All authors; (VII) \\ Final approval of manuscript: All authors. \\ Correspondence to: Kenneth P. Seastedt, MD. 1060 W. Perimeter Rd., Malcolm Grow Medical Center, Joint Base Andrews, MD 20762, USA. Email: \\ kenneth.seastedt@gmail.com, kenneth.p.seastedt.mil@mail.mil.
}

Background: Lung cancer remains the leading cause of cancer deaths in the United States, and lung cancer screening has been shown to decrease this mortality. Adherence to lung cancer screening is paramount to realize the mortality benefit, and reported adherence rates vary widely. Few reports address non-adherence to screening, and our study sought to understand the non-compliant patients in our military population.

Methods: This Institutional Review Board approved retrospective review of patients enrolled in our screening program from 2013-2019 identified patients who failed to obtain a subsequent Low Dose CT scan (LDCT) within 15 months of their prior scan. Attempts were made to contact these patients and elucidate motivations for non-adherence via telephone.

Results: Of the 242 patients enrolled, 183 (76\%) patients were adherent to the protocol. Significant predictors of non-adherence versus adherence were younger age $(\mathrm{P}=0.008)$, female sex $(\mathrm{P}=0.005)$, and enlisted officer rank $(\mathrm{P}=0.03)$. There was no difference with regards to race, smoking status, pack-years, negative screens, lung-RADS level, or nodule size. 31 (52\%) non-adherent patients were contacted, and $24(77 \%)$ reported their reason for non-adherence was lack of follow-up for a LDCT. Twenty (64\%) were interested in re-enrollment. Of the total screening cohort, 15 interventions were performed, with lung cancer identified in $5(2 \%)$ - a $67 \%$ false positive rate. One stage IV lung cancer was found in a non-adherent patient who re-enrolled.

Conclusions: Lack of perceived contact for follow-up was expressed as the primary reason for noncompliance in our screening program. Compliance is critical to the efficacy of any screening modality, and adherence rates to lung cancer screening may be increased through improved contact with patients via multiple avenues (i.e., phone, email, and letter). There is benefit in contacting non-adherent patients as high rates of re-enrollment are possible.

Keywords: Lung cancer screening; low dose CT scan (LDCT); adherence; enrollment; smoking

Submitted May 04, 2020. Accepted for publication Aug 14, 2020.

doi: $10.21037 /$ jtd-20-1841

View this article at: http://dx.doi.org/10.21037/jtd-20-1841 


\section{Introduction}

Lung cancer remains the leading cause of cancer deaths in the United States, accounting for roughly 1 in 4 deaths from cancer in 2019 (1,2). Given the low survival rate and late presentation of the majority of lung cancer cases, efforts have been made to identify early lung cancers via screening low dose CT scan (LDCT). Detection of lung cancer earlier in the disease course allows for more effective treatment, as in the successful screening campaigns for other cancers such as cervical, breast, prostate, and colon cancer (3). Randomized clinical trials such as the National Lung Screening Trial (NLST) and the Dutch-Belgian Randomized Lung Cancer Screening Trial (NELSON trial) have demonstrated decreased mortality from lung cancer through effective screening (4-11). It is estimated that lung cancer screening can prevent greater than 12,000 premature cancer deaths yearly (12).

Lung cancer screening has been endorsed by several major U.S. medical societies in addition to the International Association for the Study of Lung Cancer (13). In 2013, the U.S. Preventative Services Task Force (USPSTF) recommended annual LDCT in asymptomatic patients aged 55-80 years with a 30 pack-year or more smoking history who are current smokers or who have quit less than 15 years ago. This recommendation requires insurers to cover screening with no cost sharing under the Affordable Care Act, and is a preventative service benefit under the Medicare program $(13,14)$. The appropriate duration of screening and the age at which screening is inappropriate is unknown $(15,16)$.

Despite these recommendations, enrollment of eligible patients for screening is exceedingly low. Only 4.4\% of eligible smokers in the U.S. received LDCT in 2015 as compared to $71.5 \%$ for breast cancer, and $62.4 \%$ for colorectal cancer $(17,18)$. Significant challenges to implementing a screening program exist, as demonstrated by the Veterans Health Administration, and screening has not been widespread (19). Once patients are enrolled, adherence to lung cancer screening protocols is paramount to realize the overall mortality benefit. Adherence to screening was high in the NLST (95\%) and NELSON trials $(90 \%)$, but varies in the literature from $51-85 \%$ outside of these major trials $(4,7,11,20,21)$. Few reports address patient non-adherence to lung cancer screening (20,22-25). Our study sought to understand the behavior of non-compliant lung cancer screening program patients in our unique Department of Defense health beneficiaries in an effort to identify areas to improve our program and adherence to lung cancer screening.

We present the following article in accordance with the SQUIRE reporting checklist (available at http://dx.doi. org/10.21037/jtd-20-1841).

\section{Methods}

The study was conducted in accordance with the Declaration of Helsinki (as revised in 2013). This performance improvement project was approved after administrative, scientific, and ethical review by the Department of Research Programs and the Walter Reed National Military Medical Center (WRNMMC) Institutional Review Board, reference number 913214. Individual consent for this retrospective analysis was waived. The WRNMMC lung cancer screening program began in 2013 through the Department of Pulmonology, and patients are enrolled only if referred to the program. Patients are confirmed that they meet the USPSTF recommendations for screening of asymptomatic adults aged 55-80 who have a 30 pack-year history, currently smoke, or have quit within the last 15 years and have the ability/willingness to have curative lung surgery (26). When enrolled, patients are educated about lung cancer, lung cancer screening, and the WRNMMC protocol for screening. Patient data is then collected including demographics, LDCT and pathology results, interventions, timing of follow-up scans, total pack-years, and smoking status. Data are stored in a password-protected customized database for our lung cancer screening program based at WRNMMC. Once data is collected, the patient is offered smoking cessation services if currently smoking, and a LDCT is ordered and scheduled during block time with radiology, which occurs one day per week. LDCT scans are reviewed by staff radiologists who are encouraged to report results using the lung-RADS protocol (27). Results are then monitored by the program manager and discussed with a staff Pulmonologist. The next screening CT scan or intervention is then planned based on the findings and radiologist recommendations, and the patient is contacted via phone to schedule a follow-up LDCT or intervention. Patients are notified via automated letter and phone call 6 weeks before a followup scan is needed by the program manager or smoking cessation counselor. Patients who miss scheduled CT scans are contacted by phone where possible. To be considered adherent to the protocol, patients have to be no more than 3 months past due for their scheduled LDCT. 
Table 1 Topics for review with non-adherent patients

Perception of in-office education regarding importance of screening

Any patient reservations regarding screening

Any perceived barriers to obtaining a low dose CT

Preferred method of contact

Reason for non-adherence

Desire for re-enrollment

Recommendations for improvement for the protocol and patient experience

All patients enrolled in the program from inception in 2013 until June 2019 who still met eligibility criteria for lung cancer screening were reviewed for adherence with the protocol. Those patients who met non-adherence were reviewed and attempts were made to contact these patients by phone. For patients contacted, semi-structured interviews were undertaken to discuss all topics listed in Table 1, and were conducted by a single provider. Patients were made aware their responses were voluntary and in no way would affect their healthcare, and questions were asked in an open-ended fashion to obtain the desired information and avoid leading patient responses. All responses to the topics in Table 1 were immediately documented after each phone interview.

\section{Statistical analysis}

Individual categorical differences between patients who were adherent and non-adherent were initially assessed using Fisher's exact test. To assess differences in continuous variables between adherent and non-adherent individuals, the Exact Wilcoxon Rank Sum test was used. A logistic regression model was used to provide adjusted estimates for multiple covariates. Alpha was set at $\mathrm{P} \leq 0.05$ for all analyses, and all analyses were completed in R 3.6.1 (R Core Team, Vienna, Austria).

\section{Results}

Demographic features of the 242 patients enrolled in our Lung Cancer Screening program can be found in Table 2. Most patients were older (median age: 67 years), ma1e (69\%), and Caucasian (58\%), 183 (76\%) patients were adherent to the screening protocol. Table 2 provides statistical tests for the association between individual features and adherence. These analyses revealed significant differences between the adherent and non-adherent groups for age $(\mathrm{P}=0.008)$, sex $(\mathrm{P}=0.005)$, and rank $(\mathrm{P}=0.03)$, with younger, female, and enlisted patients in the non-adherent group. There was no difference between the adherent and non-adherent groups with regards to race, smoking status, pack-years, negative screens, lung-RADS level, or nodule size.

An adjusted model using age, race, sex, negative screens, smoking status, and rank confirmed significant effects of age (OR, 1.43, 95\% CI, 1.03-2.01, $\mathrm{P}=0.03)$ and sex $(\mathrm{OR}, 2.57$, $95 \%$ CI, 1.36-4.87, P=0.003), but no effect of rank. Because of the identified age and sex differences, we also examined how adherence varied with age in males and females separately. Here, we observed that while men tended to increase adherence as they aged (OR, 2.38, 95\% CI, 1.494.10), women tended to have numerically decreasing but statistically consistent adherence across the lifespan (OR, $0.71,95 \%$ CI, 0.40-1.25).

Of the $59(24 \%)$ non-adherent patients, they were overdue for LDCT by an average of 29 months, and 31 $(52 \%)$ were able to be contacted via phone (Table 3). Of the patients contacted, $93 \%$ stated their education about the importance of screening and the protocol in office were adequate, and no patients reported having any reservations about undergoing and continuing lung cancer screening. 24 (77\%) reported their reason for not following through with lung cancer screening was that they were not contacted to schedule a follow-up LDCT, 4 (13\%) reported screening was not a priority as they were dealing with more pressing medical issues, and $3(10 \%)$ reported they were continuing screening at another facility. Twenty (64\%) reported they were interested in being re-enrolled after being contacted. When asked about their preferred method of contact, 14 (45\%) preferred phone calls, 13 (42\%) preferred emails, 3 (10\%) had no preference, and 1 (3.2\%) preferred letters.

Of the entire cohort, 15 interventions have been performed as a result of screening, including bronchoalveolar lavage, bronchoscopy with or without biopsy, percutaneous biopsy and/or surgical resection. Lung cancer was identified in 5 cases ( $2 \%$ of total study population), and there was a $67 \%(10 / 15)$ rate of negative or non-diagnostic intervention after a purportedly concerning LDCT study (Table 4). Of the identified cancers, 1 (20\%) was stage I, 1 (20\%) was stage II, and 2 (40\%) were stage III according to the American Joint Committee on Cancer $8^{\text {th }}$ edition (28). One of the patients who was re-enrolled 
Table 2 Demographics of adherent versus non-adherent patients

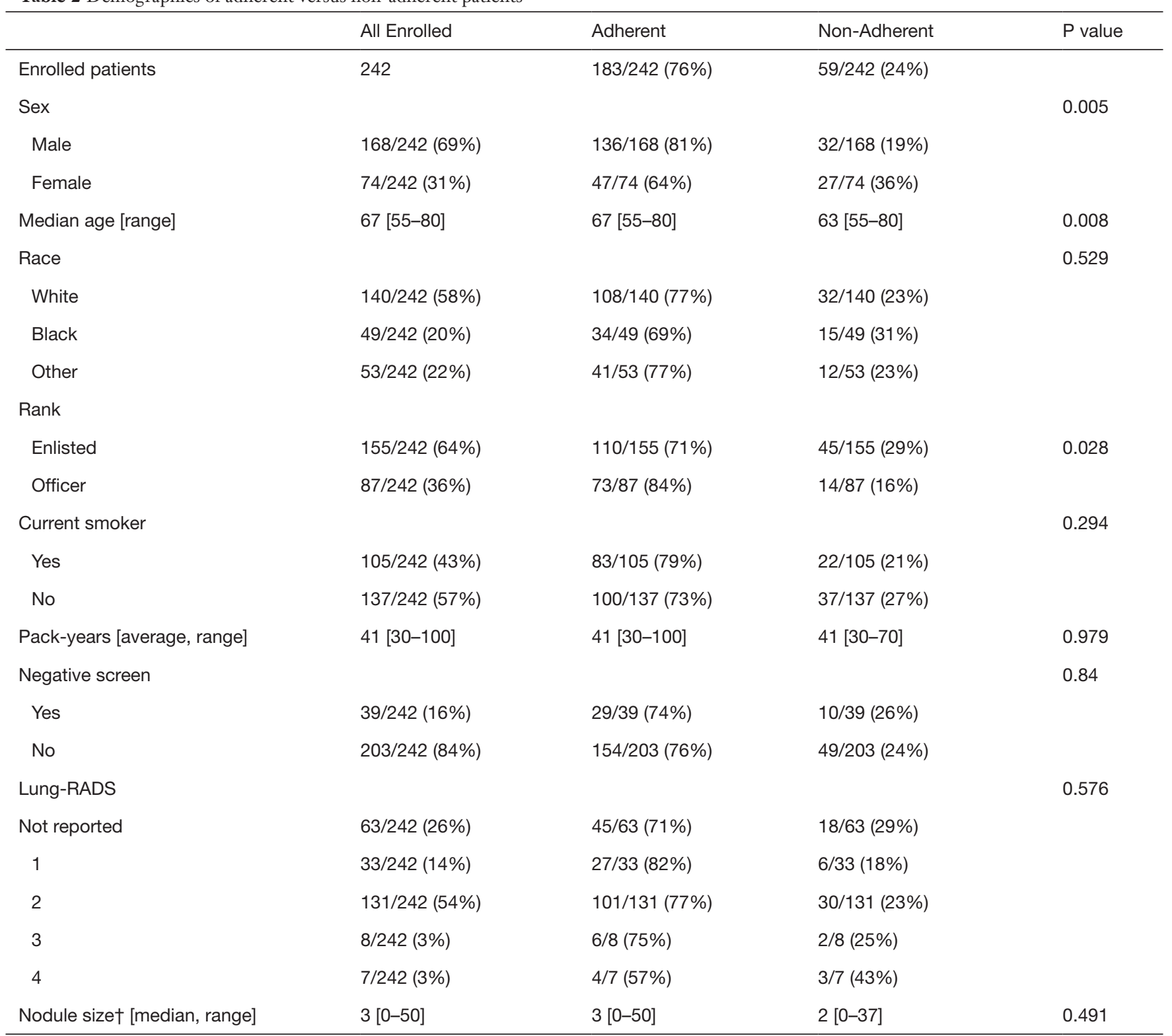

$\dagger$, measured in millimeters.

from the non-adherent group was found to have squamous cell carcinoma of the lung with metastases on her updated screening LDCT. The stage I cancer was cT1aN0 in a 65 -year-old male with a 30 pack-year smoking history and was treated with stereotactic body radiation therapy per the patient's preference despite surgical recommendations. The stage II cancer was stage IIB adenocarcinoma (pT2aN1) in a 59-year-old female with a 30 pack-year history, treated with surgery followed by adjuvant chemotherapy. Regarding the stage III cancers, one was stage IIIA adenocarcinoma in a 64-year-old female with an 80 pack-year smoking history and treated with surgery, adjuvant chemoradiotherapy (CRT), and maintenance durvalumab. The other stage III cancer is a stage IIIB limited small cell lung cancer in a 
Table 3 Non-adherent patient characteristics

\begin{tabular}{|c|c|}
\hline & $\mathrm{N}$ \\
\hline Months overdue [average, range] & 29 [15-54] \\
\hline Able to be contacted & $31(52 \%)$ \\
\hline \multicolumn{2}{|l|}{ Adequate education } \\
\hline Yes & $29(93 \%)$ \\
\hline No & $2(7 \%)$ \\
\hline \multicolumn{2}{|l|}{ Reservations about LCS } \\
\hline Yes & $0(0 \%)$ \\
\hline No & $31(100 \%)$ \\
\hline \multicolumn{2}{|l|}{ Reason for non-adherence } \\
\hline Not contacted to follow-up & $24(77 \%)$ \\
\hline Other medical issues & $4(13 \%)$ \\
\hline Being followed elsewhere & $3(10 \%)$ \\
\hline Refusal to follow-up & $0(0 \%)$ \\
\hline \multicolumn{2}{|l|}{ Desire to re-enroll } \\
\hline Yes & $20(64 \%)$ \\
\hline No & $11(36 \%)$ \\
\hline \multicolumn{2}{|l|}{ Preferred method of contact } \\
\hline Phone & $14(45 \%)$ \\
\hline Email & $13(42 \%)$ \\
\hline No preference & $3(10 \%)$ \\
\hline Letter & $1(3.2 \%)$ \\
\hline
\end{tabular}

63 -year-old female with a 60 pack-year smoking history, who is currently undergoing CRT.

\section{Conclusions}

Lung cancer survival rates are significantly higher with early stage disease, and outcomes dramatically decline with increasing stage. Five-year survival is below $25 \%$ for Stage III, and only $5 \%$ for patients with distant disease-the most common presentation $(2,29)$. Through identification of lung cancers earlier in their disease course, lung cancer screening with LDCT has been shown to reduce lung cancer mortality by $20-41 \%$ in the NLST and NELSON trials (4-11). In the NELSON trial, 59\% of lung cancers were identified as stage I, compared to only $16 \%$ identified at the same stage currently in the U.S. $(2,7,11)$. This stage
Table 4 Lung cancer screening interventions

\begin{tabular}{|c|c|c|}
\hline & $\mathrm{N}$ & Patient details \\
\hline Interventions Performed & $15(6 \%)$ & \\
\hline False positive & $10(67 \%)$ & \\
\hline Cancers Identified & $5(2 \%)$ & \\
\hline Stage I & $1(20 \%)$ & $\begin{array}{l}\text { Estimated Stage IA: } \\
\text { cT1aN0, SBRT }\end{array}$ \\
\hline Stage II & $1(20 \%)$ & $\begin{array}{l}\text { Stage IIB: pT2aN1, } \\
\text { surgery, adjuvant } \\
\text { chemotherapy }\end{array}$ \\
\hline \multirow[t]{2}{*}{ Stage III } & $2(40 \%)$ & $\begin{array}{l}\text { Stage IIIA: pT1bN2, } \\
\text { surgery, adjuvant } \\
\text { CRT, maintenance } \\
\text { durvalumab }\end{array}$ \\
\hline & & $\begin{array}{l}\text { Stage IIIB: T3N2 } \\
\text { Limited Small Cell Lung } \\
\text { Cancer, CRT }\end{array}$ \\
\hline Stage IV & $1(20 \%)$ & $\begin{array}{l}\text { Updated screen } \\
\text { found Squamous } \\
\text { Cell Carcinoma with } \\
\text { metastatic disease. } \\
\text { Patient was re-enrolled } \\
\text { after non-adherence }\end{array}$ \\
\hline
\end{tabular}

migration allowed a surgical intervention with curative intent rate of $68 \%$ in the treatment arm versus only $25 \%$ in the control group. The majority of cancers $(60 \%)$ found during our screening program were amenable to surgical intervention. This is consistent with findings in the NSLT and NELSON trials, which is in contrast to the majority of patients presenting nationally with advanced lung cancer. Given that surgical intervention remains the mainstay of curative therapy for lung cancer, these results highlight the central importance of thoracic surgeons in the screening and treatment of lung cancer.

As demonstrated, high adherence rates are critical to realize the mortality benefit of lung cancer screening, and the adherence rate was $76 \%$ within our program. One cancer was found in a patient who was re-enrolled 54 months after her index scan, and was unfortunately found to have squamous cell lung cancer with distant disease on her updated screening LDCT. Her case illustrates the critical importance of efforts to improve patient adherence to a screening program. Our adherence rate is higher than reported rates for breast and colon cancer screening and consistent with other rates reported in the 
community, however not as high as the major trials. Little is known about patient motivations for non-adherence to screening, and it has been suggested that lack of awareness or understanding of screening benefits, screening fatigue, the risks involved with screening, false-negative and falsepositive results, radiation exposure, anxiety, complications from workup interventions, financial costs, patient refusal to follow-up, inability to contact the patient, male gender, and proximity to referral centers are all factors influencing adherence (20,22-25). Of the non-adherent patients that were able to be contacted from our program, $77 \%$ reported their reasoning for non-adherence was lack of contact to follow-up and schedule their next scan. This was despite $93 \%$ stating they had adequate education about the program and protocol, and $100 \%$ stating they had no reservations about continuing with screening. We suspect this lack of patient follow-up could be secondary to an inability to contact the patients reliably via phone. Our non-adherent patients reported phone calls were not the unanimously preferred method of contact, especially in light of increased telemarketing causing many patients to ignore unknown phone numbers. Many (42\%) preferred email communication, and stated their emails remained stable despite changing addresses and phone numbers. Once contacted, the majority (64\%) wished to be re-enrolled and scheduled for a follow-up LDCT. Although we were able to demonstrate that non-adherent patients were more likely to be younger, female, and in an enlisted rank, we believe all patients should be targeted to maintain enrollment. Based on these results, we hypothesize that additional administrative support to increase contact via multiple avenues (phone, email, and letter) with all enrolled patients regardless of age, sex, race, rank or smoking history will continue to improve and maintain adherence to screening. This is especially important in those patients who become non-adherent as many can be recaptured. Commercially available software programs that provide comprehensive tracking and reporting systems to decrease administrative burden are another consideration to augment established lung cancer screening programs.

In addition to non-adherence to lung cancer screening is the issue of underutilization of screening. The military health system serves over 9 million beneficiaries, with WRNMMC being the nation's largest joint military medical center (30). Certainly more patients would qualify for our program than the currently enrolled 242 patients. This underutilization of LDCT for screening within the military health system parallels the nation as a whole with lack of adequate implementation seen nationwide in the general U.S. population as well as the Veterans Health Administration system $(18,19)$. The slow adoption of lung cancer screening is likely multifactorial, with one possible contribution being lack of awareness or understanding regarding screening. Currently, the American Academy of Family Physicians deems there is insufficient evidence to date to recommend lung cancer screening (31). It has also been shown that protocols used to identify eligible patients vary widely in the community and that the off-guideline use of LDCT screening may be common (32). The Lung Cancer Screening Registry sponsored by the American College of Radiology has over 4,000 participating facilities and continues to grow, but there certainly are still patients and providers that may not have access to a lung cancer screening program (33).

There may be concerns about the risk/benefit ratio, as over-diagnosis and high false positive rates leading to invasive follow-up examinations have been demonstrated in the NSLT and VA populations (4,19). A study of pulmonologists' views on LDCT for lung cancer screening in 2015 reported $52.4 \%$ had a propensity for guidelineconcordant screening, $22.7 \%$ for over-screening, and $24.9 \%$ for under-screening. Pulmonologists recommending underscreening cited the harms of potential screening including false positive results, radiation exposure, unfavorable costbenefit ratio, and also cite insufficient infrastructure and personnel as barriers to implementation (34). Additionally, the collection of lung cancer screening data from records is labor-intensive and there may be a role for automated techniques to obtain this data in the future $(19,32)$. Reengaging primary and specialist physicians to educate about the benefits of screening and to improve on documentation of accurate smoking histories will improve enrollment, adherence, and any attempts to use electronic health records for lung cancer screening evaluations.

To combat the high false-positive rates associated with screening, risk prediction modeling has been proposed to identify patients for screening based on individual risk as opposed to just age and smoking exposure. Many models have been developed that have retrospectively performed better (especially the PLCOm2012 model) at risk prediction than the NSLT criteria (35). Although promising, there is no information on long term mortality reduction using these models, and these models would need to prove to be effective and user-friendly across a large population. One well-established means to increase the detection of lung cancer and to decrease the rate of false positive CT results 
is through the consistent utilization of the lung-RADS reporting system, the use of which is strongly encouraged at the facilities participating in our LDCT screening program $(13,27,36,37)$.

Regarding the potential untoward psychological effects of LDCT screening such as patient anxiety, guilt or shame, none of the non-adherent patients in our study expressed that these feelings were a subjective impediment to continued screening (38). In terms of the cost effectiveness of screening, initial analyses of the NLST data found screening to be cost-effective at $\$ 81,000$ per qualityadjusted life-year (QALY) gained, and several analyses have reported on the cost-effectiveness ratio of $\$ 100,000$ or less per QALY gained for LDCT $(39,40)$.

The results of this present study have several limitations. Firstly, there is inherent bias in semi-structured interviews from recall bias to response bias on the part of the patient, based on what they remember of their experience and also how positively they wished to respond the questions posed by the interviewer. Efforts were made to ask neutral questions, avoid leading questions, and ensure participants understood the survey would in no way affect their healthcare or relationship with their providers. Additionally, a single interviewer was used in an effort to provide a consistent format and result. Despite the inherent disadvantages of semi-structured interviews, they have been shown to provide meaningful data and insight for clinical decision making (41). Furthermore, our retrospective study not only involved a single-institution, but also our patient population is unique with regards to increased risk of smoking and occupational exposure to carcinogens, which may limit the generalizability of these results (42). Prior reports suggest lung cancer rates up to double in military veterans compared to the general U.S. population (43). Additionally, our analysis and conclusions are based off of the $52 \%$ of non-adherent patients who were able to be contacted by phone, highlighting the need for multiple accurate methods to contact patients. It is possible these patients may be getting screening in civilian institutions as $10 \%$ of those we were able to contact were being followed elsewhere.

Lung cancer screening is an important method of identifying lung cancer at early stages, allowing for earlier and more effective surgical interventions, and an associated potential for improved long-term survival. Although compliance is critical to the efficacy of any screening modality, prior studies on LDCT for lung cancer screening reveal little about reasons for lack of patient adherence. By contacting patients in our LDCT lung cancer screening protocol who were non-adherent, we determined that the majority cited lack of follow-up to schedule the next LDCT as their reason for non-adherence. Adherence rates may be increased through improved contact with patients through multiple avenues of communication (i.e., phone, email, letter), and there is benefit in reaching out to non-adherent patients as high rates of re-enrollment are possible.

\section{Acknowledgments}

The authors would like to acknowledge the assistance of Mr. Patrick Shuster, Dr. Daniel Brooks, and Ms. Adriana Martinez. This data was presented at the 2019 Eastern Cardiothoracic Surgical Society Annual Meeting in Naples, FL.

Funding: None.

\section{Footnote}

Reporting Checklist: The authors have completed the SQUIRE reporting checklist. Available at http://dx.doi. org/10.21037/jtd-20-1841

Data Sharing Statement: Available at http://dx.doi. org/10.21037/jtd-20-1841

Conflicts of Interest: All authors have completed the ICMJE uniform disclosure form (available at http://dx.doi. org/10.21037/jtd-20-1841). The authors have no conflicts of interest to declare.

Disclaimer: No authors have financial disclosures or conflicts of interest, and the views expressed are solely those of the authors and do not reflect the official policy or position of the US Army, US Navy, US Air Force, the Department of Defense, or the US Government.

Ethical Statement: The authors are accountable for all aspects of the work in ensuring that questions related to the accuracy or integrity of any part of the work are appropriately investigated and resolved. The study was conducted in accordance with the Declaration of Helsinki (as revised in 2013). The study was approved by institutional review board of Walter Reed National Military Medical Center (\#913214) and individual consent for this 
retrospective analysis was waived.

Open Access Statement: This is an Open Access article distributed in accordance with the Creative Commons Attribution-NonCommercial-NoDerivs 4.0 International License (CC BY-NC-ND 4.0), which permits the noncommercial replication and distribution of the article with the strict proviso that no changes or edits are made and the original work is properly cited (including links to both the formal publication through the relevant DOI and the license). See: https://creativecommons.org/licenses/by-nc-nd/4.0/.

\section{References}

1. Torre LA, Siegel RL, Ward EM, et al. Global Cancer Incidence and Mortality Rates and Trends--An Update. Cancer Epidemiol Biomarkers Prev 2016;25:16-27.

2. National Cancer Institute. SEER Cancer Statistics Review, 1975-2016. Accessed September 2019. Available online: https://seer.cancer.gov/csr/1975_2016/

3. Cronin KA, Lake AJ, Scott S, et al. Annual Report to the Nation on the Status of Cancer, part I: National cancer statistics. Cancer 2018;124:2785-800.

4. Aberle DR, Adams AM, Berg CD, et al. Reduced lungcancer mortality with low-dose computed tomographic screening. N Engl J Med 2011;365:395-409.

5. Aberle DR, DeMello S, Berg CD, et al. Results of the two incidence screenings in the National Lung Screening Trial. N Engl J Med 2013;369:920-31.

6. Church TR, Black WC, Aberle DR, et al. Results of initial low-dose computed tomographic screening for lung cancer. N Engl J Med 2013;368:1980-91.

7. de Koning HJ, van der Aalst CM, de Jong PA, et al. Reduced Lung-Cancer Mortality with Volume CT Screening in a Randomized Trial. N Engl J Med 2020;382:503-13.

8. Midthun DE. Screening for lung cancer. Clin Chest Med 2011;32:659-68.

9. Yousaf-Khan U, van der Aalst C, de Jong PA, et al. Risk stratification based on screening history: the NELSON lung cancer screening study. Thorax 2017;72:819-24.

10. van Klaveren RJ, Oudkerk M, Prokop M, et al. Management of lung nodules detected by volume CT scanning. N Engl J Med 2009;361:2221-9.

11. De Koning H, Van Der Aalst C, Ten Haaf K, et al. PL02.05 Effects of Volume CT Lung Cancer Screening: Mortality Results of the NELSON RandomisedControlled Population Based Trial. J Thorac Oncol
2018;13:S185.

12. Ma J, Ward EM, Smith R, et al. Annual number of lung cancer deaths potentially avertable by screening in the United States. Cancer 2013;119:1381-5.

13. National Comprehensive Cancer Network. Lung Cancer Screening (Version 1.2020). Accessed September 2019. Available online: https://www.nccn.org/professionals/ physician_gls/pdf/lung_screening.pdf

14. Centers for Medicare \& Medicaid Services. Decision Memo for Screening for Lung Cancer with Low Dose Computed Tomography (LDCT) (CAG-00439N). Accessed September 10, 2019. Available online: https:// www.cms.gov/medicare-coverage-database/details/ncadecision-memo.aspx?NCAId $=274$

15. Han SS, Ten Haaf K, Hazelton WD, et al. The impact of overdiagnosis on the selection of efficient lung cancer screening strategies. Int J Cancer 2017;140:2436-43.

16. Bach PB, Mirkin JN, Oliver TK, et al. Benefits and harms of CT screening for lung cancer: a systematic review. JAMA 2012;307:2418-29.

17. Jemal A, Fedewa SA. Lung Cancer Screening With LowDose Computed Tomography in the United States-2010 to 2015. JAMA Oncol 2017;3:1278-81.

18. Richards TB, Doria-Rose VP, Soman A, et al. Lung Cancer Screening Inconsistent With U.S. Preventive Services Task Force Recommendations. Am J Prev Med 2019;56:66-73.

19. Kinsinger LS, Anderson C, Kim J, et al. Implementation of Lung Cancer Screening in the Veterans Health Administration. JAMA Intern Med 2017;177:399-406.

20. Montes U, Seijo LM, Campo A, et al. Factors determining early adherence to a lung cancer screening protocol. Eur Respir J 2007;30:532-7.

21. Cattaneo SM 2nd, Meisenberg BR, Geronimo MCM, et al. Lung Cancer Screening in the Community Setting. Ann Thorac Surg 2018;105:1627-32.

22. Wiener RS. Balancing the benefits and harms of lowdose computed tomography screening for lung cancer: Medicare's options for coverage. Ann Intern Med 2014;161:445-6.

23. Detterbeck FC. Overdiagnosis during lung cancer screening: is it an overemphasised, underappreciated, or tangential issue? Thorax 2014;69:407-8.

24. Rampinelli C, De Marco P, Origgi D, et al. Exposure to low dose computed tomography for lung cancer screening and risk of cancer: secondary analysis of trial data and riskbenefit analysis. BMJ 2017;356:j347.

25. Alshora S, McKee BJ, Regis SM, et al. Adherence to 
Radiology Recommendations in a Clinical CT Lung Screening Program. J Am Coll Radiol 2018;15:282-6.

26. Moyer VA. Screening for lung cancer: U.S. Preventive Services Task Force recommendation statement. Ann Intern Med 2014;160:330-8.

27. American College of Radiology. Lung-RADSTM Version 1.0 Assessment Categories; 2014. Accessed September 17, 2019. Available online: https://www.acr.org/-/ media/ACR/Files/RADS/Lung-RADS/LungRADS_ AssessmentCategories.pdf? la1/4en

28. Detterbeck FC, Boffa DJ, Kim AW, et al. The Eighth Edition Lung Cancer Stage Classification. Chest 2017;151:193-203.

29. Rami-Porta R, Ball D, Crowley J, et al. The IASLC Lung Cancer Staging Project: proposals for the revision of the $\mathrm{T}$ descriptors in the forthcoming (seventh) edition of the TNM classification for lung cancer. J Thorac Oncol 2007;2:593-602.

30. Lin J, Kamamia C, Brown D, et al. Survival among Lung Cancer Patients in the U.S. Military Health System: A Comparison with the SEER Population. Cancer Epidemiol Biomarkers Prev 2018;27:673-9.

31. American Academy of Family Physicians. Clinical Preventive Service Recommendation: Lung Cancer. Accessed September 17. Available online: https://www. aafp.org/patient-care/clinicalrecommendations/all/lungcancer.html

32. Gould MK, Sakoda LC, Ritzwoller DP, et al. Monitoring Lung Cancer Screening Use and Outcomes at Four Cancer Research Network Sites. Ann Am Thorac Soc 2017;14:1827-35.

33. American College of Radiology. Lung Cancer Screening Registry. Accessed September 17 2019. Available online: https://www.acr.org/-/media/ACR/Files/Registries/LCSR/ LCSR-Participants.pdf

Cite this article as: Seastedt KP, Luca MJ, Antevil JL, Browning RF, Mullenix PS, Reoma JL, McKay SA. Patient motivations for non-adherence to lung cancer screening in a military population. J Thorac Dis 2020;12(10):5916-5924. doi: $10.21037 /$ jtd-20-1841
34. Iaccarino JM, Clark J, Bolton R, et al. A National Survey of Pulmonologists' Views on Low-Dose Computed Tomography Screening for Lung Cancer. Ann Am Thorac Soc 2015;12:1667-75.

35. Ten Haaf K, Jeon J, Tammemägi MC, et al. Risk prediction models for selection of lung cancer screening candidates: A retrospective validation study. PLoS Med 2017;14:e1002277.

36. Pinsky PF, Gierada DS, Black W, et al. Performance of Lung-RADS in the National Lung Screening Trial: a retrospective assessment. Ann Intern Med 2015;162:485-91.

37. McKee BJ, Regis SM, McKee AB, et al. Performance of ACR Lung-RADS in a Clinical CT Lung Screening Program. J Am Coll Radiol 2016;13:R25-9.

38. Harris RP, Sheridan SL, Lewis CL, et al. The harms of screening: a proposed taxonomy and application to lung cancer screening. JAMA Intern Med 2014;174:281-5.

39. Black WC, Gareen IF, Soneji SS, et al. Cost-effectiveness of CT screening in the National Lung Screening Trial. N Engl J Med 2014;371:1793-802.

40. Puggina A, Broumas A, Ricciardi W, et al. Costeffectiveness of screening for lung cancer with low-dose computed tomography: a systematic literature review. Eur J Public Health 2016;26:168-75.

41. DeJonckheere M, Vaughn LM. Semistructured interviewing in primary care research: a balance of relationship and rigour. Fam Med Community Health 2019; 7:e000057.

42. Krefft SD, Rose CS, Nawaz S, et al. Deployment-Related Lung Disorders. Fed Pract 2015;32:24S-31S.

43. Harris RE, Hebert JR, Wynder EL. Cancer risk in male veterans utilizing the Veterans Administration medical system. Cancer 1989;64:1160-8. 